\title{
Исследование устойчивости метастабильных эпитаксиальных слоев GeSn к термическим воздействиям
}

\author{
(C) В.П. Мартовицкий ${ }^{+}$Ю.Г. Садофьев ${ }^{+\ddagger}$, А.В. Клековкин ${ }^{+}$, В.В. Сарайкин ${ }^{*}$, И.С. Васильевский \\ + Физический институт им. П.Н. Лебедева Российской академии наук, \\ 119991 Москва, Россия \\ * Национальный исследовательский институт фризических проблем им. Ф.В. Лукина, \\ 124460 Зеленоград, Москва, Россия \\ ‡ Национальный исследовательский ядерный университет „Московский инженерно-физический институт“, \\ 115409 Москва, Россия \\ E-mail: aklekovkinbox@gmail.com
}

(Получена 27 апреля 2016 г. Принята к печати 10 мая 2016 г.)

На виртуальной подложке (001) Si/Ge была выращена структура, состоящая из пяти упругонапряженных метастабильных слоев GeSn толщиной 200 нм каждый, разделенных прослойками из германия толщиной 20 нм. Мольная доля олова в слоях GeSn имела следующие значения: 0.005, 0.034, 0.047, 0.072 и 0.10. После роста структура подвергалась термическому отжигу в течение 2 мин при температуре $400^{\circ} \mathrm{C}$. Показано, что в процессе отжига наряду с пластической релаксацией происходит фазовый распад сплава GeSn, который начинается еще до завершения процесса пластической релаксации. Структурная деградация слоев $\mathrm{GeSn}$ возрастала с ростом концентрации олова, которая накапливалась на поверхности структуры в виде аморфного слоя.

\section{1. Введение}

Твердые растворы $\mathrm{Ge}_{1-x} \mathrm{Sn}_{x}$ перспективны в качестве материалов с прямой структурой запрещенной зоны на основе элементарных полупроводников с кристаллической решеткой алмаза. В последние годы проведен ряд исследований, результатом которых стало наблюдение прямых в пространстве импульсов оптических переходов в спектрах фотолюминесценции вплоть до комнатной температуры [1,2]. В [3] показана возможность получения лазерной генерации в слоях $\mathrm{Ge}_{0.88} \mathrm{Sn}_{0.12}$, выращенных на кремниевой пластине с буферным слоем германия. Когерентное излучение было получено при оптической накачке с плотностью мощности на уровне 400 кВт/см² при температурах до $90 \mathrm{~K}$. При всей значимости данного результата для фундаментальной науки перспективность его для практического применения пока не ясна.

Серое олово $(\alpha-\mathrm{Sn})$ является полуметаллом с перекрытием зоны проводимости и валентной зоны, оцениваемым величиной 0.4 эВ. Простая интерполяция зонных структур германия и серого олова предсказывает, что ненапряженный твердый раствор $\mathrm{Ge}_{1-x} \mathrm{Sn}_{x}$ должен иметь прямую структуру запрещенной зоны в интервале мольной доли олова $\sim(0.2-0.65)$. В указанном интервале составов твердого раствора ширина запрещенной зоны $E_{G}$ должна изменяться от 0.55 до 0 эВ [4]. Расчеты показывают [5,6], что напряжения двухосного растяжения, в том числе гетероэпитаксиальные, также приводят к уменьшению ширины запрещенной зоны, причем влияние напряжений на ширину прямой запрещенной зоны значительно сильнее. Переход от непрямозонного материала к прямозонному может наблюдаться при сравнительно малой мольной доле олова, $x \approx 0.02$.
Имеющиеся экспериментальные данные дают величину $x \approx 0.09$ для реализации указанного перехода $[7,8]$.

Однако малое значение термодинамически равновесного предела растворимости $\alpha$-Sn в германии $(x=0.005)$, больший радиуса атома олова $(0.158$ нм $)$, по сравнению с атомом германия $(0.139 \mathrm{Hм})$ и низкое значение поверхностной свободной энергии $\mathrm{Sn}$, обусловливают склонность к сегрегации олова при эпитаксиальном выращивании твердых растворов системы GeSn.

Из сказанного следует, что твердые растворы GeSn с мольной долей олова, представляющей практический интерес, могут быть получены только в далеких от термодинамического равновесия условиях. Они с неизбежностью будут метастабильными, с тенденцией к фазовому распаду при внешних воздействиях. В дополнение к этому при гетероэпитаксии слоев GeSn на инородных подложках возникают встроенные механические напряжения. Они модифицируют зонную структуру материала и могут влиять на протекание процессов пластической релаксации и фазового распада GeSn.

\section{2. Эксперимент}

Выращивание GeSn проводили на установке молекулярно-пучковой эпитаксии (МПЭ) „Катунь“, укомплектованной двумя электронно-лучевыми испарителями для кремния и германия, а также двумя молекулярными источниками типа ячейки Кнудсена. Эти последние источники использовали для соиспарения сурьмы в качестве сурфактанта при выращивании буферных слоев германия толщиной 1 мкм [9] и для испарения олова при выращивании слоев GeSn. Дифрактометр быстрых электронов (RHEED) позволял контролировать все стадии 
ростового процесса in situ. Периодическое измерение скорости испарения кремния и германия проводили с помощью кварцевого резонатора, встроенного в ростовую камеру. Свойства полученных эпитаксиальных слоев контролировали с помощью рентгеновской дифрактометрии на дифрактометре Panalytical X'pert Pro MRD Extended, масс-спектрометрии вторичных ионов (SIMS) на установке IMS-4f фирмы CAMECA, атомно-силовой микроскопии (AFM) с использованием прибора Solver P47H фирмы NT-MDT и оже-спектроскопии. Отжиг выращенных образцов проводили в установке RTA Modular в газовой среде особо чистого азота.

Ранее нами было показано, что относительно толстые ( 0.5 мкм) слои $\mathrm{GeSn}$ с мольной долей олова до 0.075, выращенные на кремнии с релаксированным буферным слоем германия в качестве виртуальной подложки, не проявляли признаков пластической релаксации в процессе роста. Однако даже кратковременный отжиг таких структур приводил как к частичной пластической релаксации, так и к выходу олова на поверхность структуры [10]. Минимальная температура отжига, приводящая к началу этих процессов, зависела от величины мольной доли олова в слоях $\mathrm{GeSn}$.

Данная работа посвящена изучению устойчивости пересыщенного твердого раствора GeSn для определения допустимых температур термообработки эпитаксиальных структур при изготовлении полупроводниковых приборов на их основе. Для уточнения характера протекающих процессов пластической релаксации и фазового распада в тонких пленках GeSn с различным содержанием олова нами была выращена многослойная структура $\mathrm{Ge} / \mathrm{GeSn}$ (образец S050). Поверх релаксированного буферного слоя германия толщиной 1 мкм, выращенного на пластине кремния ориентации (001), были выращены 5 слоев $\mathrm{GeSn}\left(\mathrm{GeSn}_{(1)}-\mathrm{GeSn}_{(5)}\right)$ номинальной толщиной 200 нм каждый, разделенные прослойками из германия толщиной 20 нм. Мольную долю олова увеличивали по мере удаления от германиевого буфера. Для этого температуру молекулярной ячейки олова изменяли в пределах $T_{\mathrm{Sn}}=920-1000^{\circ} \mathrm{C}$ с шагом $20^{\circ} \mathrm{C}$ при переходе от слоя к слою. В дальнейшем описании результатов эксперимента слой GeSn с меньшим номером соответствует его расположению ближе к буферному слою германия. Скорость роста эпитаксиальных слоев $\mathrm{GeSn}$ поддерживали на уровне $\sim 3$ нм/мин. Структуру завершал покровный слой германия толщиной 20 нм. Температура эпитаксии была близка к $150^{\circ} \mathrm{C}$. Из наблюдений картин RHEED следует, что при столь низких температурах роста все выращенные слои $\mathrm{GeSn}$ являются монокристаллическими. По мере повышения мольной доли олова развиваются признаки фасетирования на поверхности растущего слоя, которые исчезают при осаждении прослоек германия. Среднеквадратичная шероховатость поверхности, соответствующая слою с максимальным из используемых в данном эксперименте содержанием олова в твердом растворе, находилась на уровне $\mathrm{rms} \approx(2-3)$ нм, тогда как та же величина для поверхности буферного слоя германия близка к 0.7 нм [9].

Рентгеноструктурные исследования проводили с первичным монохроматором, состоящим из комбинации рентгеновского зеркала и 4-кристального монохроматора $4 \times \operatorname{Ge}(220)$ с расходимостью $12^{\prime \prime}$, и третьим кристаллом-анализатором $3 \times \mathrm{Ge}(220)$. Для определения параметра решетки слоев перпендикулярно к ростовой поверхности использовали рефлекс (004), а для определения параметра решетки в плоскости срастания рефлекс (224). Дифрактометр был отъюстирован по монокристаллу кремния КДБ-12 с параметром решетки $a=5.43105 \AA$ таким образом, чтобы ошибка в положении максимума пика (004) после третьего кристаллаанализатора не превышала $0.0001^{\circ}$. Фрагмент двумерной области вблизи рефлекса (004) был получен при записи 85 кривых качания (004) с третьим кристалломанализатором в режиме $(2 \theta-\omega)$-сканирования, с поворотом образца на $\Delta \omega=0.005^{\circ}$ при записи следующей кривой. Все операции производились в автоматическом режиме. Затем с помощью программы Panalytical Epitaxy все эти кривые были интегрированы в двумерную область в обратном пространстве.

\section{3. Результаты эксперимента}

На рис. 1 приведен фрагмент двумерной области вблизи рефлекса (004) подложки $\mathrm{Si}$, буферного слоя $\mathrm{Ge}$ и трех слоев $\mathrm{GeSn}$ с наименьшими мольными долями олова $\left(\mathrm{GeSn}_{(1)}-\mathrm{GeSn}_{(3)}\right)$. Положение каждого пика по оси $Q_{y}$ обратного пространства определяется величиной брэгговского угла дифракции $\left(\theta_{\mathrm{B}}\right)[11]$ : $Q_{y}=\sin \theta_{\mathrm{B}} \cdot \cos \left(\theta_{\mathrm{B}}-\omega\right) \cdot 10^{4}$, где $\omega-$ угол падения рентгеновского луча на образец, величина которого для симметричного рефлекса (004) близка к значению угла Брэгга. Для $\mathrm{Si} Q_{y}=5674$ e.o.p. (единиц обратной решетки, reciprocal lattice units - r.l.u.), для Ge $Q_{y}=5455$ e.o.p. Большая величина несоответствия между параметрами решетки буферного слоя и подложки $(4.18 \%)$ приводит не только к полной пластической релаксации напряжений несоответствия в буферном слое, но и к уширению его пика по горизонтальной оси. Положение центра пика (или его уширение) по оси $Q_{x}$ соответствует величине разориентации кристаллических плоскостей от (001) [8]: $Q_{x}=\sin \theta_{\mathrm{B}} \cdot \sin \left(\theta_{\mathrm{B}}-\omega\right) \cdot 10^{4}$. Центр пика $\mathrm{Si}$ имеет значение $Q_{x}=-11.2$ e.o.p., для которого угол падения $\omega=34.642^{\circ}$ больше величины брэгговского угла $\theta_{\mathrm{B}}=34.564^{\circ}$ на $0.078^{\circ}$. Уширение пика германия $\Delta\left(Q_{x}\right)= \pm 12.5$ e.o.p. на рефлексе $(004)$ соответствует разориентации его кристаллических плоскостей на $\Delta \omega= \pm 0.085^{\circ}$ от плоскости (001) Si. Все слои $\mathrm{GeSn}$ наследуют эту величину разориентации. Поэтому сам буферный слой германия и растущие на нем слои $\mathrm{GeSn}$ фактически представляют собой текстуру из-за высокой плотности дислокаций, имеющих тенденцию к формированию малоугловых границ. Отметим также 


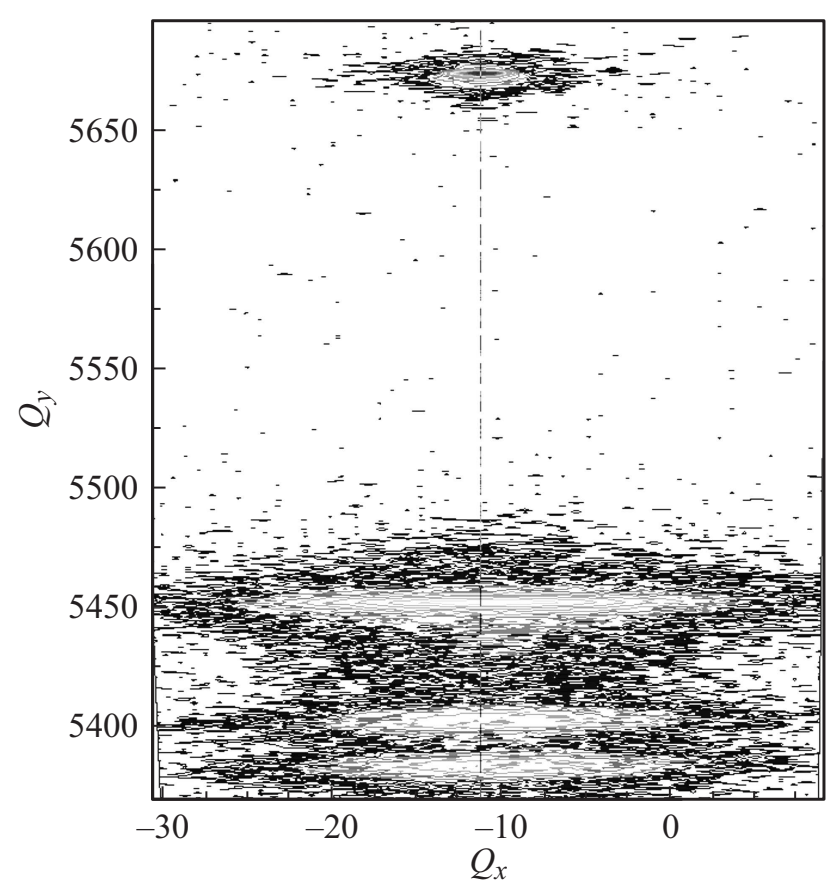

Рис. 1. Фрагмент двумерной области вблизи рефлекса (004) образца S050, включающий пик кремния (вверху), пик буферного слоя $\mathrm{Ge}$ при $Q_{y}=5455$ e.o.p., и трех ближайших к нему слоев $\mathrm{GeSn}$. Слой $\mathrm{GeSn}_{(1)}$ с минимальной мольной долей олова $\left(T_{\mathrm{Sn}}=920^{\circ} \mathrm{C}\right)$ лишь слегка отделен от пика буферного слоя германия. Два последующих слоя, $\mathrm{GeSn}_{(2)}, \mathrm{GeSn}_{(3)}\left(T_{\mathrm{Sn}}=940\right.$ и $\left.960^{\circ} \mathrm{C}\right)$, хорошо различимы в нижней части рисунка.

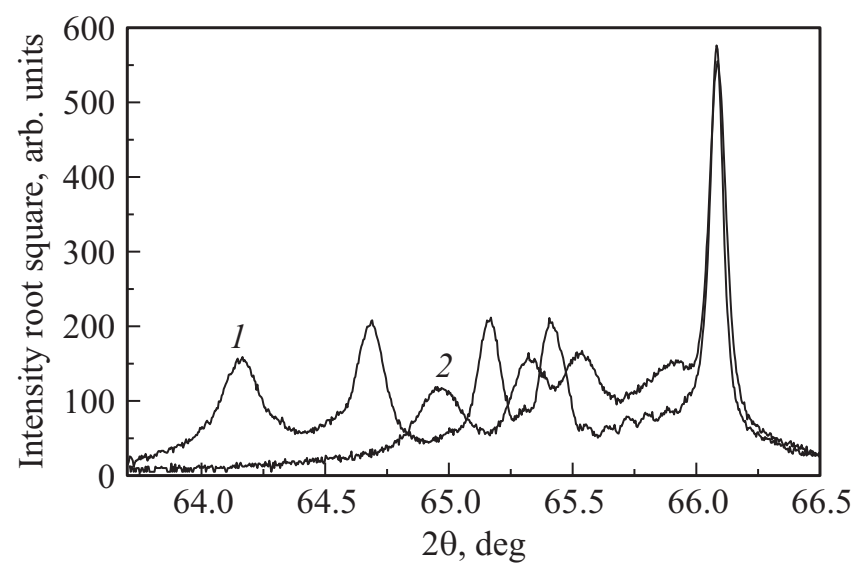

Рис. 2. Кривые $(2 \theta-\omega)$ сканирования на рефлексе (004) в квадратичном масштабе с третьим кристаллом-анализатором, записанные после выращивания образца $\mathrm{S} 050$ (1) и после отжига в течение 2 мин при $400^{\circ} \mathrm{C}(2)$.

повышенное диффузное рассеяние вблизи пика германия и слоев $\mathrm{GeSn}$.

Запись обычной кривой качания с широкой приемной щелью на детекторе на таком текстурированном образце не дает хорошего разрешения между пиками эпитаксиальных слоев GeSn. Поэтому на рис. 2 в квадратичном масштабе приведены кривые $(2 \theta-\omega)$ - сканирования на рефлексе (004) с третьим кристалломанализатором. Кривая 1 была записана непосредственно после получения образца, а кривая 2 - после отжига при $400^{\circ} \mathrm{C}$ в течение 2 мин. Пик кремния из-за высокой интенсивности исключен на рис. 2, хотя реально прописывался для контроля юстировки дифрактометра. По положениям максимумов на этих кривых можно определить значения периода кристаллической решетки в направлении роста слоев, $a_{\perp}$, для буферного слоя германия и всех пяти слоев GeSn. На кривой 1 рис. 2 между наиболее интенсивным пиком от буферного слоя германия и ближайшим к нему хорошо разрешенным пиком от слоя $\mathrm{GeSn}_{(2)}$ видны сателлиты, по угловому расстоянию между которыми можно определить толщину этого слоя. Слой $\mathrm{GeSn}_{(1)}$ из-за малой концентрации олова не разделяется с пиком буферного слоя германия, и значение параметра решетки для него было получено путем подгонки экспериментальной и расчетной кривых качания. Это, с учетом сложной конструкции структуры в целом, может привести к заметной погрешности в определении мольной доли олова в нем из данных рентгеновских измерений.

На рис. 2 видны три принципиальных различия между кривыми 1 и 2, до отжига и после отжига образца, а именно: 1) полное отсутствие после отжига пика от слоя $\mathrm{GeSn}_{(5)}$, выращенного при максимальной из использованных в данной работе температуре источника олова $\left.\left(T_{\mathrm{sn}}=1000^{\circ} \mathrm{C}\right) ; 2\right)$ сдвиг трех других пиков слоев $\mathrm{GeSn}_{(4)}-\mathrm{GeSn}_{(2)}$ в сторону бо́льших углов, что соответствует понижению концентрации олова в них; 3) лучшее разделение с пиком германия пика от слоя $\mathrm{GeSn}_{(1)}$ из-за возросшей концентрации олова в нем.

Для точного определения мольной доли олова в слоях GeSn необходима запись асимметричных рефлексов (224), которые приведены на рис. 3. По положениям максимумов пиков на асимметричном рефлексе (224) были получены значения параметров решетки в плоскости срастания $\left(a_{\|}\right)$. Для нахождения величины мольной

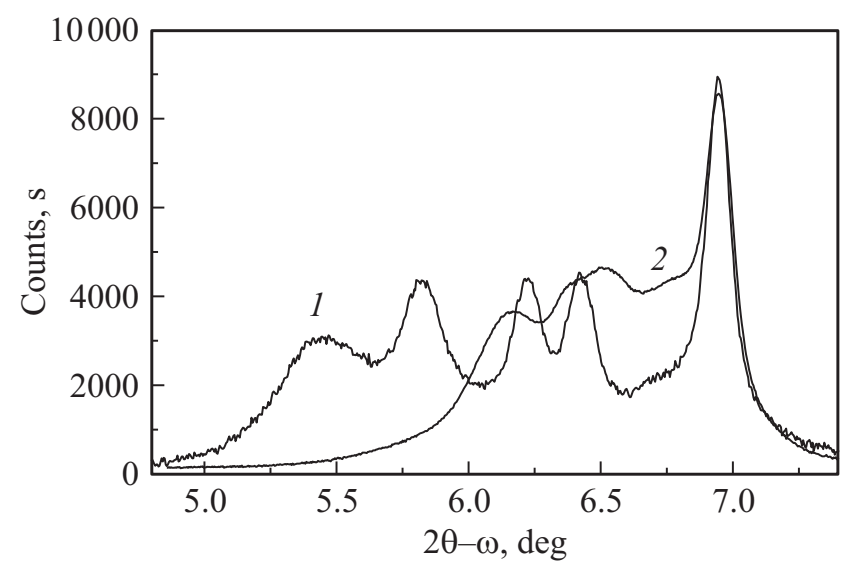

Рис. 3. Кривые $(2 \theta-\omega)$-сканирования в квадратичном масштабе на рефлексе (224) образца S050, записанные до (1) и после отжига в течение 2 мин при $400^{\circ} \mathrm{C}(2)$. 
Релаксированные параметры кристаллических решеток GeSn отдельных слоев структуры и значения мольной доли олова в них

\begin{tabular}{c|c|c|c|c}
\hline \multirow{2}{*}{ Слой } & \multicolumn{2}{|c|}{ После роста } & \multicolumn{2}{|c}{ После отжига $\left(400^{\circ} \mathrm{C}, 2\right.$ мин $)$} \\
\cline { 2 - 5 } & Мольная доля $\mathrm{Sn}$ & Релаксация, \% & Мольная доля $\mathrm{Sn}$ & Релаксация, \% \\
\hline $\mathrm{Ge}-$ буфер $_{\mathrm{GeSn}}$ & 0 & 103.5 & 0 & 103.5 \\
$\mathrm{GeSn}_{(2)}$ & $\sim 0.005$ & 0 & 0.008 & 0 \\
$\mathrm{GeSn}_{(3)}$ & 0.034 & -9.9 & 0.033 & 69.9 \\
$\mathrm{GeSn}_{(4)}$ & 0.047 & 2.7 & 0.04 & 0 \\
$\mathrm{GeSn}_{(5)}$ & 0.072 & -1.7 & 0.066 & 30.5 \\
\end{tabular}

доли олова в слоях нужно определить релаксированные значения параметра решетки $\mathrm{GeSn}$; они вычисляются с использованием коэффициента Пуассона $v$, значение которого также зависит от концентрации олова. Поэтому определение состава делалось в процессе двух последовательных итераций: сначала с приблизительным значением коэффициента Пуассона, а затем с его уточненным значением. Для германия $v=0.273$, для олова $v=0.300$. Период кристаллической решетки $\alpha$-Sn был взят равным $6.491 \AA$.

Для расчетов значений релаксированных параметров кристаллических решеток слоев GeSn было использовано уравнение, в котором $a_{\perp}$ и $a_{\|}$обозначают периоды кристаллических решеток материалов в направлении роста и в плоскости поверхности структуры соответственно [12]:

$$
\left(\frac{\Delta a}{a_{s}}\right)_{\text {relax }}=\frac{1-v}{1+v}\left(\frac{\Delta a}{a_{s}}\right)_{\perp}+\frac{2 v}{1+v}\left(\frac{\Delta a}{a_{s}}\right)_{\|} .
$$

Для буферного слоя германия в расчетах в качестве значения $a_{s}$ использовали значение параметра решетки кремния, тогда как для слоев $\mathrm{GeSn}$ значение $a_{s}$ равно параметру виртуальной подложки германия в плоскости срастания, $a_{\| \mathrm{Ge}}$.

Значение параметра решетки буферного слоя германия в плоскости срастания, $a_{\| \mathrm{Ge}}=5.6650 \AA$, больше, чем в перпендикулярном направлении, $a_{\perp}=5.6513 \AA$, из-за различия коэффициентов термического расширения германия, $\alpha_{\mathrm{Ge}}(300 \mathrm{~K})=5.7 \cdot 10^{-6}{ }^{\circ} \mathrm{C}^{-1}$, и кремния, $\alpha_{\mathrm{Si}}(300 \mathrm{~K})=2.6 \cdot 10^{-6}{ }^{\circ} \mathrm{C}^{-1}$. Слой германия, полностью пластически релаксированный при температуре эпитаксии, испытывает действие двуосных напряжений растяжения при охлаждении до комнатной температуры. Формально это приводит к тому, что релаксация буферного слоя германия становится $>100 \%$.

До отжига образца с возрастанием концентрации олова в эпитаксиальном слое наблюдается возрастание значения параметра решетки перпендикулярно к ростовой поверхности, тогда как в плоскости срастания параметр решетки $\mathrm{GeSn}$ совпадает или даже может быть слегка меньше параметра решетки буферного слоя германия. Это означает не только отсутствие релаксации напряжений несоответствия при эпитаксии, но и присутствие повышенной концентрации вакансий в виде каких-либо устойчивых комплексов, обладающих сжимающим действием на значение параметра решетки. Процент релаксации для германия рассчитывался как $\operatorname{rel}_{\mathrm{Ge}}=100\left(a_{\mathrm{Ge} \|}-a_{\mathrm{Si}}\right) /\left(a_{\mathrm{Ge} \mathrm{relax}}-a_{\mathrm{Si}}\right)$, где $a_{\mathrm{Si}}=5.43105 \AA$. Для каждого слоя $\mathrm{GeSn}$ вместо параметра кремния берется значение параметра решетки в плоскости срастания предыдущего слоя, например $\operatorname{rel}_{\mathrm{GeSn}_{(3)}}=100\left(a_{\mathrm{GeSn}_{(3)} \|}-a_{\left.\mathrm{GeSn}_{(2)} \|\right)} /\left(a_{\mathrm{GeSn}_{(3)}}\right.\right.$ relax $\left.-a_{\mathrm{GeSn}_{(2)} \|}\right)$.

На основании расчетов релаксированных параметров кристаллических решеток GeSn отдельных слоев структуры определены значения мольной доли олова в них (см. таблицу). Эти величины изменяются как 0.005 , $0.034,0.047,0.072$ и 0.10 по мере удаления слоев $\mathrm{GeSn}$ от буферного слоя германия. Из известных справочных данных для зависимости плотности насыщенных паров олова от температуры следует, что изменение плотности паров на один порядок величины в интервале температур, использованных в наших экспериментах, требует изменения температуры источника олова на $110^{\circ} \mathrm{C}$. Таким образом, обнаруженное методом XRD изменение мольной доли олова в 20 раз при изменении температуры молекулярной ячейки олова на $80^{\circ} \mathrm{C}$ представляется завышенным. Ожидаемая величина этого изменения не должна быть более чем 7-кратной. Наибольшее относительное изменение мольной доли олова (примерно в 7 раз) наблюдается при переходе от слоя $\mathrm{GeSn}_{(1)}$ к слою $\mathrm{GeSn}_{(2)}$. При малых количествах олова в слое система частично отталкивает атомы $\mathrm{Sn}$, которые увеличивают упругую энергию несоответствия. При больших же концентрациях они входят в структуру слоя, но вместе с точечными дефектами - вакансиями. Таким образом, кристаллическое качество слоев деградирует с увеличением содержания олова в них, что особенно заметно для слоя с максимальной для данного эксперимента мольной долей олова на уровне $x \sim 0.1$, рентгеновский пик которого заметно уширен (рис. 3). Тенденция к уширению наблюдается и для предшествующего пика, соответствующего слою с мольной долей олова $x=0.072$.

Профиль распределения олова в структуре после роста, измеренный методом SIMS при использовании первичных ионов $\mathrm{O}_{2}^{+}$с энергией 8 кэВ, приведен на рис. 4 (кривая 1) для ионов олова $\mathrm{Sn}^{+}$. Все пять ступеней $\mathrm{GeSn}$ с различным содержанием олова в них 


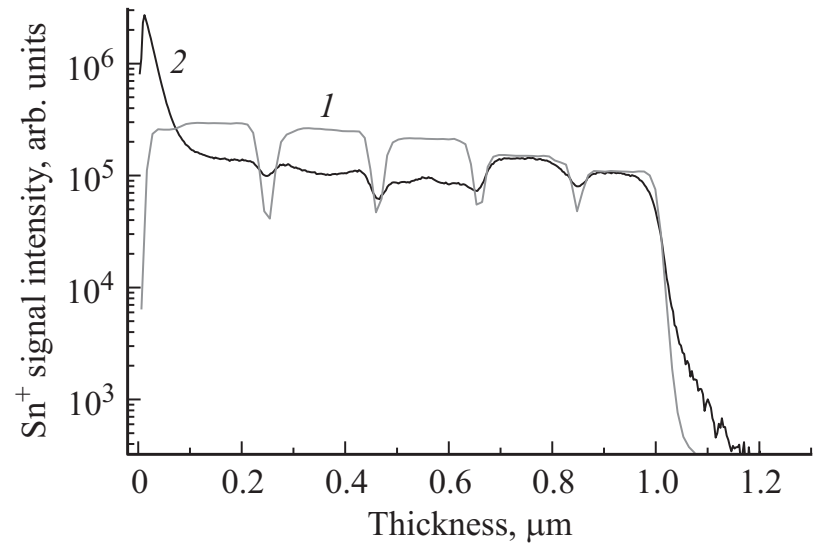

Рис. 4. Профили распределения олова в структуре S050, содержащей пять слоев GeSn c различной мольной долей олова, разделенных тонкими прослойками из германия. $1-$ после выращивания образца; 2 - после отжига структуры в течение 2 мин при температуре $400^{\circ} \mathrm{C}$. Вид кривых соответствует интенсивности сигнала вторичных ионов $\mathrm{Sn}^{+}$при измерении профилей.

выявляются отчетливо, как и разделительные прослойки германия малой толщины (20 нм). При этом, в отличие от данных XRD, относительное изменение амплитуды сигнала вторичных ионов $\mathrm{Sn}^{+}$между соседними слоями практически одинаковое для всех 5 слоев структуры. Расхождение данных методов XRD и SIMS для слоев с наименьшей мольной долей олова может быть обусловлено различием физических явлений, лежащих в основе этих методов. XRD использует для определения мольной доли олова степень отклонения параметров кристаллической решетки GeSn от того же для германия, т.е. XRD регистрирует те атомы олова, которые вошли в кристаллическую решетку твердого раствора $\mathrm{GeSn}$, что приводит к увеличению объема кристаллической решетки по сравнению с германиевой матрицей. SIMS же чувствителен к общей концентрации олова в слое, в том числе и к атомам, находящимся в межузельных положениях или формирующих дефектно-преципитатные комплексы в твердом растворе. В частности, широко известно явление геттерирования примесей дислокациями [13]. В нашем случае, при выращивании $\mathrm{GeSn}$ на релаксированном буферном слое германия толщиной 1 мкм плотность прорастающих дислокаций не может быть $<1 \cdot 10^{6} \mathrm{~cm}^{-2}$. Это создает предпосылки для формирования дислокационно-преципитатных комплексов, в результате чего только часть олова, введенного в слой, входит в кристаллическую ячейку GeSn в областях, свободных от дислокаций. Емкость этого канала оттока атомов олова ограничена величиной плотности дислокаций. Поэтому эффект формирования преципитатнодислокационных комплексов проявляется в максимальной степени в XRD-экспериментах при использовании малой плотности потоков олова, сопоставимых с емкостью канала оттока этих атомов на дислокации. Это, по нашему мнению, и является причиной различия данных XRD и SIMS по концентрации олова в слоях с наименьшими из использованных мольными долями олова $\left(\mathrm{GeSn}_{(1)}\right.$ и $\left.\mathrm{GeSn}_{(2)}\right)$.

После отжига структурные параметры слоев изменяются самым драматичным образом (см. кривые 2 на рис. 2,3, а также таблицу). Кроме полного отсутствия пика от слоя $\mathrm{GeSn}_{(5)}$ с исходно максимальной концентрацией олова, одновременно наблюдается понижение концентрации олова в слоях $\mathrm{GeSn}_{(4)}$ и $\mathrm{GeSn}_{(3)}$. Влияние отжига на слои с низкой мольной долей олова $\left(\mathrm{GeSn}_{(2)}\right.$ и $\left.\mathrm{GeSn}_{(1)}\right)$ не столь значительно.

Профили SIMS (рис. 4, кривая 2) дают более детальную информацию о перераспределении олова в структуре в результате отжига. Термообработка в минимальной степени затрагивает слои $\mathrm{GeSn}_{(1)}$ и $\mathrm{GeSn}_{(2)}$. Однако и для этих слоев наблюдается процесс диффузионного размытия профиля распределения олова, приводящий к заметному изменению глубины модуляции состава при переходе от слоев $\mathrm{GeSn}$ к разделительным прослойкам германия. Как показано на профилях, олово диффундирует в обоих направлениях, но в глубь структуры диффузия идет в значительно меньшей степени из-за того, что при диффузии олова в глубь кристалла увеличивается упругая энергия несоответствия. Именно из-за перекоса скоростей диффузии на внешнюю поверхность и в глубь кристалла мы и наблюдаем увеличение концентрации олова только в самом первом эпитаксиальном слое, при этом в слоях $\mathrm{GeSn}_{(2)}$ и $\mathrm{GeSn}_{(3)}$ обеднение оловом заметно меньше, чем в слое $\mathrm{GeSn}_{(4)}$ и тем более в слое $\mathrm{GeSn}_{(5)}$. Это приводит к заметному огрублению микрорельефа поверхности, регистрируемому с помощью атомно-силовой микроскопии. Сила эффекта зависит от количества олова, имеющегося в структуре (от мольной доли олова и толщины слоя $\mathrm{GeSn}$ ). Среднеквадратичная шероховатость поверхности $\mathrm{rms}$ для слоев $\mathrm{GeSn}$ толщиной 0.5 мкм и с мольной долей олова 0.075 в результате отжига может изменяться от 2 до 40 нм [7]. Отжиг образца не приводит к полному исчезновению германиевых прослоек между слоями твердых раствоpoв GeSn. Это означает, что диффузия олова идет преимущественно по границам зерен, образовавшимся в виртуальной подложке германия и прорастающим в слои GeSn.

\section{4. Заключение}

Приведенные выше данные показывают, что с увеличением мольной доли $\mathrm{Sn}$ в твердых растворах $\mathrm{GeSn}$ повышается склонность к фазовому распаду, сопровождающемуся обеднением слоев оловом. При отжиге слоев $\mathrm{GeSn}$ происходят два конкурирующих процесса, а именно фазовый распад твердого раствора с последующим выходом олова на поверхность структуры в основном по границам зерен, а также пластическая релаксация твердого раствора без изменения его состава. Домини- 
рование того или иного процесса определяется скачком мольной доли олова между соседними слоями. Фазовый распад начинается задолго до завершения пластической релаксации упругонапряженных эпитаксиальных слоев $\mathrm{GeSn}$, а сама пластическая релаксация не оказывает особенного влияния на процесс распада твердого раствора и диффузии олова. По нашему мнению, это связано с повышенной концентрацией дефектов в слоях GeSn, выращенных на виртуальной подложке Ge.

Работа поддержана грантами Российского фонда фундаментальных исследований (№ 13-02-00680 А и 13-02-12164 офи_м).

\section{Список литературы}

[1] R. Ragan, H.A. Atwater. Appl. Phys. Lett., 77, 3418 (2000).

[2] G. Grzybowski, R.T. Beeler, L. Jiang, D.J. Smith, J. Kouvetakis, J. Menendez. Appl. Phys. Lett., 101, 072105 (2012).

[3] S. Wirthst, R. Geiger, N. von den Driesch, G. Mussler, T. Stoica, S. Mantl, Z. Ikonic, M. Luysberg, S. Chiussi, J. M. Hartmann, H. Sigg, J. Faist, D. Buca, D. Grützmacher. Nature Photonics, 9, 88 (2015).

[4] S. Ogus, W. Paul, T.F. Deutsch, B.-Y. Tsaur, D.V. Murphy. Appl. Phys. Lett., 43, 848 (1983).

[5] R.A. Sofer, L. Friedman. Superlatt. Microstr., 14, 189 (1993).

[6] O. Gurdal, P. Desjardins, J.R.A. Carlsson, N. Taylor, H.H. Radamson, J.-E. Sundgren, J.E. Greene. J. Appl. Phys., 83, 162 (1998).

[7] G. He, H.A. Atwater. Phys. Rev. Lett., 79, 1937 (1997).

[8] J. Mathews, R.T. Beeler, J. Tolle, C. Xu, R. Roucka, J. Kouvetakis, J. Menéndez. Appl. Phys. Lett., 97, 221912 (2010).

[9] Ю.Г. Садофьев, В.П. Мартовицкий, М.А. Базалевский. Изв. РАН. Сер. физ., 78, 47 (2014).

[10] Ю.Г. Садофьев, В.П. Мартовицкий, М.А. Базалевский, А.В. Клековкин, Д.В. Аверьянов, И.С. Васильевский. ФТП, 49, 128 (2015).

[11] N. Herres, F. Fuchs, J. Schmitzetal. Phys. Rev. B, 53, 15688 (1996).

[12] S.N.G. Chu, A.T. Macrander, K.E. Strege, W.D. Johnston. J. Appl. Phys., 57, 249 (1985).

[13] К. Рейви. Дефекты и примеси в полупроводниковом кремнии (М., Мир, 1984).

Редактор Л.В. Шаронова

\section{Thermal stability of $\mathrm{Ge} / \mathrm{GeSn}$ nanostructures grown by MBE on (001) $\mathrm{Si} / \mathrm{Ge}$ virtual wafers}

\author{
V.P. Martovitsky ${ }^{+}$, Yu.G. Sadofyev ${ }^{+\ddagger}$, \\ A.V. Klekovkin ${ }^{+}$, V.V. Saraikin*, I.S. Vasil'evskii \\ + Lebedev Physical Institute, \\ Russian Academy of Sciences, \\ 119991 Moscow, Russia \\ * Lukin Research Institute of Physical Problems, \\ 124460 Zelenograd, Moscow, Russia \\ ‡National Research Nuclear University „MEPhl“, \\ 115409 Moscow, Russia
}

\begin{abstract}
A stack of five metastable 200-nm-thick elastically strained GeSn epitaxial layers separated by 20-nm-thick Ge spacers was grown on (001) $\mathrm{Si} / \mathrm{Ge}$ virtual substrate by MBE. The molar fraction of $\mathrm{Sn}$ in different layers varied as $0.005,0.034,0.047,0.072$ and 0.10 , increasing with the layer distance from the Ge buffer. After growth the structure was subjected to thermal annealing for $2 \mathrm{~min}$ at the temperature $400^{\circ} \mathrm{C}$. The phase separation of the $\mathrm{GeSn}$ alloy during postgrowth annealing takes place along with plastic relaxation. The phase separation begins well before the completion of the plastic relaxation process. The degree of phase separation at a given annealing temperature depends strongly on the Sn content in the GeSn alloy. The Sn released from the decomposed GeSn alloy predominantly accumulates as an amorphous layer on the surface of the sample.
\end{abstract}

